\title{
Pancreatic Exocrine Insufficiency after Pancreatic Surgery Detected by Tubeless Testing
}

\author{
Masao Yagi, ${ }^{*}$ Kouichi Shimizu, Tetsuo Hashimoto, Ryouhei IzUmi, \\ Takukazu NAGAKAwA, Kouichi MiWA, and Itsuo MIYAZAKI \\ Department of Surgery II, School of Medicine, \\ Kanazawa University, Kanazawa 920, Japan
}

(Received January 10, 1994)

\begin{abstract}
Summary The pancreatic function diagnostant (PFD test) and fecal chymotrypsin test were performed simultaneously in patients after pancreatic surgery, and findings for both tests were analyzed. The $p$ aminobenzoic acid recovery rate in the PFD test and the chymotrypsin activity in stools decreased after pancreatectomy, particularly after extended radical pancreaticoduodenectomy. Chymotrypsin activity in stools, measured by the fecal chymotrypsin test after extended radical pancreaticoduodenectomy, was significantly lower than the normal level. The D-xylose output in the extended radical group was significantly $(p<$ $0.05)$ lower than that following conventional pancreaticoduodenectomy. The results allow the conclusion that the absorption factor must be taken into account when evaluating the pancreatic exocrine function by the PFD test.
\end{abstract}

Key Words: postoperative pancreatic exocrine insufficiency, pancreatic function diagnostant, fecal chymotrypsin test, D-xylose test

Pancreaticoduodenectomy is classified into two types. One is an extended radical pancreaticoduodenectomy, which includes massive regional pancreatectomy [1], resection of the nervous plexus of the superior mesenteric artery, retroperitoneal lymph node dissection, and sleeve resection of portal vein [2]; and the other is a conventional pancreaticoduodenectomy. Some patients develop malnutrition following extended radical pancreaticoduodenectomy, due to pancreatic insufficiency and abnormal absorption [3]. It is, therefore, important for the nutritional management of these patients to measure postoperative pancreatic exocrine insufficiency [4] and to compare pancreatic exocrine function with

*To whom correspondence should be addressed. 
respect to changes in absorption after these two types of pancreaticoduodenectomy have been performed.

There are two tubeless methods that can be used to detect pancreatic exocrine insufficiency in postoperative patients. One is the pancreatic function diagnostant (PFD test, Bentiromide test) [5], and the other is the fecal chymotrypsin test (FCT) [6]. The FCT directly measures chymotrypsin activity remaining in the stool, whereas the PFD test evaluates the chymotrypsin activity in the duodenum according to rate of recovery of $p$-aminobenzoic acid (PABA) from the urine. PABA released from peptides is absorbed through the small intestine, and thus it is important to evaluate the absorption of PABA. It is possible to evaluate such absorption by the D-xylose test, which is a kind of clinical absorption test. In this paper we report our experience using PFD, FCT, and D-xylose test in patients after pancreatic surgery.

\section{MATERIALS AND METHODS}

A total of 57 patients were evaluated, and of these 32 underwent pancreaticoduodenectomy for pancreaticoduodenal malignancy; 12 underwent side-toside pancreaticojejunostomy for chronic pancreatitis; 8 underwent distal pancreatectomy for cancer of the pancreatic tail or chronic pancreatitis; and 5 underwent total pancreatectomy for pancreatic cancer. Pancreatic function tests were performed in patients within 10 months to 1 year after surgery. Nutritional control was favorable, and there were no signs of cancer recurrence. Fifty-three healthy volunteers were chosen as controls.

The patients undergoing pancreaticoduodenectomy were divided into 2 groups according to the extent of resection. One group consisted of patients who underwent extended radical pancreaticoduodenectomy [1] (extended radical group, $n=10$ ) which included about $60 \%$ pancreatectomy, resection of the nervous plexus of the superior mesenteric artery, retroperitoneal lymph node dissection, and sleeve resection of the portal vein. The other group consisted of patients who underwent conventional pancreaticoduodenectomy ( $40 \%$ pancreatectomy) without resection of the nervous plexus or lymph node dissection (conventional group, $n=$ 22).

After complete withdrawal of all gastrointestinal drug treatment for 2 days, patients and volunteers fasted overnight. Five grams of D-xylose and one ampoule $(0.5 \mathrm{~g})$ of bentiromide containing $169 \mathrm{mg}$ of PABA (Eisai Co. Ltd., Tokyo) were administered orally at the same time. Only oral intake of water was allowed, and urine was collected during the first $6 \mathrm{~h}$ following administration of the D-xylose and bentiromide. The levels of D-xylose in urine were expressed as total output (grams). The PABA levels in urine were measured by the DACA ( $p$-dimethyl amino cinnamaldehyde) method [7], and the results were expressed as a percentage of the administered dose. Stools were collected the following day and fecal chymotrypsin activity was measured with a fecal chymotrypsin test kit (Boehringer 
Mannheim, Mannheim) [6]. Fecal chymotrypsin activity was expressed as international units (U) in $1 \mathrm{~g}$ of feces.

The findings in each group were expressed as the mean $\pm \mathrm{SD}$. Student's $t$-test was used to determine the significance of the difference between the means, and differences with $p$-values of less than 0.05 were taken as significant.

\section{RESULTS}

The recovery rate of $\mathrm{PABA}$ in the extended group was $31 \pm 14 \%$, and this rate was significantly $(p<0.05)$ lower than the $50 \pm 17 \%$ found in the conventional group. The D-xylose output from the extended radical group was $0.83 \pm 0.15 \mathrm{~g}$, and that from conventional group was $1.42 \pm 0.19 \mathrm{~g}$. This difference was significant $(p<0.05)$.

The recovery rate of PABA was $54 \pm 9 \%$ and $66 \pm 13 \%$ in the patients undergoing side-to-side pancreaticojejunostomy and distal pancreatectomy, respectively. There were no significant differences between the healthy controls, the side-to-side pancreaticojejunostomy group, and distal pancreatectomy group. In patients undergoing total pancreatectomy the recovery rate of PABA was $20 \pm 12 \%$, and the D-xylose output was $0.93 \pm 0.22 \mathrm{~g}$; both values were significantly lower than those for healthy controls (Table 1).

Table 1. PABA recovery rate from urine in the PFD test and D-xylose output in a $5-\mathrm{g}$ D-xylose test within 10 months to 1 year after surgery.

\begin{tabular}{lccc}
\hline \multicolumn{1}{c}{ Surgical procedure } & $n$ & $\begin{array}{c}\text { PABA recovery } \\
\text { rate }(\%)\end{array}$ & $\begin{array}{c}\text { D-Xylose output } \\
(\mathrm{g})\end{array}$ \\
\hline Healthy control & 53 & $79 \pm 12$ & $1.68 \pm 0.26$ \\
Extended radical pancreaticoduodenectomy & 10 & $31 \pm 14^{*}$ & $0.83 \pm 0.15^{*}$ \\
Conventional pancreaticoduodenectomy & 22 & $50 \pm 17$ & $1.42 \pm 0.19^{* *}$ \\
Side-to-side pancreaticojejunostomy & 12 & $54 \pm 9$ & $1.62 \pm 0.35$ \\
Distal pancreatectomy & 8 & $66 \pm 13$ & $1.60 \pm 0.32$ \\
Total pancreatectomy & 5 & $20 \pm 12^{*}$ & $0.93 \pm 0.22^{*}$ \\
\hline
\end{tabular}

Values are mean $\pm \mathrm{SD},{ }^{*} p<0.05$ vs. healthy control, ${ }^{* *} p<0.05$ vs. extended radical pancreaticoduodenectomy.

Table 2. Chymotrypsin activity in the fecal chymotrypsin test within 10 months to 1 year after surgery.

\begin{tabular}{lcc}
\hline \multicolumn{1}{c}{ Surgical procedure } & $n$ & $\begin{array}{c}\text { Chymotrypsin activity } \\
(\mathrm{U} / \mathrm{g})\end{array}$ \\
\hline Healthy control & 53 & $17.6 \pm 7.8$ \\
Extended radical pancreaticoduodenectomy & 10 & $6.0 \pm 3.1^{*}$ \\
Conventional pancreaticoduodenectomy & 22 & $9.8 \pm 6.0$ \\
Side-to-side pancreaticojejunostomy & 12 & $12.5 \pm 6.4$ \\
Distal pancreatectomy & 8 & $18.7 \pm 6.3$ \\
Total pancreatectomy & 5 & $0.2 \pm 0.2^{* *}$ \\
\hline
\end{tabular}

Values are mean $\pm \mathrm{SD},{ }^{*} p<0.05$ vs. healthy control, ${ }^{* *} p<0.05$ vs. any of the other groups.

Vol. 16, No. 3, 1994 
Chymotrypsin activity in the stool, measured by FCT after extended radical pancreaticoduodenectomy, was $6.0 \pm 3.1 \mathrm{U} / \mathrm{g}$, significantly lower than the healthy control level $(17.6 \pm 7.8 \mathrm{U} / \mathrm{g})$. After side-to-side pancreaticojejunostomy, chymotrypsin activity was $12.5 \pm 5.4 \mathrm{U} / \mathrm{g}$; and after distal pancreatectomy it was $18.7 \pm 6.3$ $\mathrm{U} / \mathrm{g}$. There were no significant differences between these two values and healthy control levels. In the group undergoing total pancreatectomy, FCT was $0.2 \pm 0.2$ $\mathrm{U} / \mathrm{g}$, a value significantly lower than that for the other groups (Table 2).

\section{DISCUSSION}

The pancreozymin-secretin test was once considered to be the most reliable of the methods available to measure pancreatic exocrine function. This method required a tube to be inserted into the duodenum or jejunum that had been anastomosed to the pancreatic duct. It is currently practical to perform a tubeless test such as the PFD test or FCT in postoperative patients. The PFD test measures the chymotrypsin activity in the duodenum from the recovery rate of PABA from the urine, and the FCT directly measures the chymotrypsin activity remaining in the stool. In the present study, the recovery rates of PABA in the PFD test after total pancreatectomy were above zero. The results suggest the existence of extrapancreatic trypsin or chymotrypsin activity in the intestine of these patients, possibly originating from paneth cells [8] or some bacteria [9]. This extrapancreatic protease activity is one factor that must be considered when evaluating the findings of the PFD test. Abnormal absorption is another factor that needs to be considered, because PABA peptide is absorbed through the small intestine. The average recovery rate of PABA from urine by the PFD test decreased after pancreaticoduodenectomy, particularly after extended radical pancreaticoduodenectomy. The absorbance of D-xylose by the small intestine after extended radical pancreaticoduodenectomy was extremely low and was significantly lower than that seen after conventional pancreaticoduodenectomy. This finding suggests that an absorption disorder may affect the recovery rate of PABA in the PFD test.

When D-xylose and PFD tests were carried out simultaneously, it was found that the dose of D-xylose did not interfere with PABA absorption even if PABA and D-xylose were simultaneously administered orally $[10,11]$. We have reported that, in the presence of an absorption disorder, the recovery rates of PABA were lower than normal and that the PFD test findings improved with an improvement in absorption. In other words, the amount of PABA absorbed must be corrected for in the PFD test depending on the absorption [12]. Bornschein [13] has also reported that the test-specificity of the PFD test improves with the combined use of PABA peptide and D-xylose. A predicted PABA excretion rate can be estimated based on the rate of $\mathrm{D}$-xylose output. The difference in PABA recovery rate between the measured and predicted values was defined as the pancreatic maldigestion ratio [12]. We suggest that the absorption abnormalities seen with the PFD 
test may be corrected for by the D-xylose output when evaluating pancreatic exocrine function in patients with absorption disorders.

\section{REFERENCES}

1. Fortner, G. (1973): Regional resection of cancer of the pancreas; A new surgical approach. Surgery, 73, 303-320.

2. Nagakawa, T., Kurachi, M., Konishi, K., and Miyazaki, I. (1982): Translateral retroperitoneal approach in radical surgery for pancreatic carcinoma. Jpn. J. Surg., 12, 229-233.

3. Yagi, M., Tomita, K., Onoda, H., Takamori, M., Takano, N., Takeshita, Y., Konishi, K., Nagakawa, T., and Miyazaki, I. (1988): Diges. Absorp., 11, 71-73 (in Japanese).

4. Takano, T., Ichiba, Y., Fujii, Y., Kodama, O., and Dohi, K. (1988): Clinical and experimental study of pancreatic exocrine function after pancreaticoduodenectomy for periampullary carcinoma. Surg. Gynecol. Obstet., 166, 200-206.

5. Hosoda, S., Kashima, K., Bamba, T., Kinugasa, K., Nakaki, T., and Masuda, M. (1979): PFD, a new test of exocrine pancreatic function using BT-PABA (bentiromide), in Pancreatic Function Diagnostant, ed. by Masuda, M., Igakushoin, Tokyo, pp. 27-37.

6. Kasper, P., Moller, G., and Wahlefeld, A. (1984): New photometric assay for chymotrypsin in stool. Clin. Chem., 30, 1753-1757.

7. Miyagawa, K., Nakajima, Y., Matsuno, S., Noto, N., Saito, Y., and Sato, T. (1979): PFD test on postoperative patients, in Pancreatic Function Diagnostant, ed. by Masuda, M., Igakushoin, Tokyo, pp. 55-59.

8. Bohe, M., Borgström, A., Lindström, C., and Ohlsson, K. (1984): Trypsin-like immunoreactivity in human paneth cells. Digestion, 30, 271-275.

9. Kakigi, M. (1985): Comparison of properties of Streptococcus faecalis derived from root canal and oral flora. J. Osaka Odontol. Soc., 48, 569-580 (in Japanese).

10. Stradley, R., Stern, R., and Heinhold, N. (1979): A method for the simultaneous evaluation of exocrine pancreatic function and absorptive function in dogs. Am. J. Vet. Res., 40, $1201-1205$.

11. Stradley, R., Rogers, W., and Sherding, R. (1981): The bentiromide: xylose test: Past, present and future. Calif. Vet., 9, 29-32.

12. Yagi, M., Onoda, H., Takamori, M., Watanabe, T., Konishi, K., Nagakawa, T., Miwa, K., and Miyazaki, I. (1988): Clinical significance of PFD and fecal chymotrypsin test in postoperative pancreatic exocrine insufficiency. Jpn. J. Gastroenterol., 85, 66-71 (in Japanese).

13. Bornschein, W. (1985): Verbesserung der Spezifitat des PABA-Tests (PFT) durch kombinierte PABA-Peptide/D-Xylose-Gebe und serumkinetische Messung? Z. Gastroenterol., 23, 247-256. 